\title{
Linear ensemble-coding in midbrain superior colliculus specifies the saccade kinematics
}

\author{
A. J. van Opstal • H. H. L. M. Goossens
}

Received: 9 October 2007 / Accepted: 21 January 2008

(C) The Author(s) 2008

\begin{abstract}
Recently, we proposed an ensemble-coding scheme of the midbrain superior colliculus (SC) in which, during a saccade, each spike emitted by each recruited SC neuron contributes a fixed minivector to the gaze-control motor output. The size and direction of this 'spike vector' depend exclusively on a cell's location within the SC motor map (Goossens and Van Opstal, in J Neurophysiol 95: 2326-2341, 2006). According to this simple scheme, the planned saccade trajectory results from instantaneous linear summation of all spike vectors across the motor map. In our simulations with this model, the brainstem saccade generator was simplified by a linear feedback system, rendering the total model (which has only three free parameters) essentially linear. Interestingly, when this scheme was applied to actually recorded spike trains from 139 saccade-related SC neurons, measured during thousands of eye movements to single visual targets, straight saccades resulted with the correct velocity profiles and nonlinear kinematic relations ('main sequence properties' and 'component stretching'). Hence, we concluded that the kinematic nonlinearity of saccades resides in the spatial-temporal distribution of SC activity, rather than in the brainstem burst generator. The latter is generally assumed in models of the saccadic system. Here we analyze how this behaviour might emerge from this simple
\end{abstract}

A. J. van Opstal $(\varangle)$

Department of Biophysics, Donders Institute for Neuroscience,

Radboud University Nijmegen, Geert Grooteplein 21,

6525 EZ Nijmegen, The Netherlands

e-mail: j.vanopstal@science.ru.nl

URL: http://www.mbfys.ru.nl/ johnvo

A. J. van Opstal · H. H. L. M. Goossens

Department of Biophysics, Donders Institute for Neuroscience,

Radboud University Nijmegen Medical Center,

Geert Grooteplein 21, 6525 EZ Nijmegen, The Netherlands scheme. In addition, we will show new experimental evidence in support of the proposed mechanism.

Keywords Saccades - Spatial accuracy - Population coding $\cdot$ Nonlinearity $\cdot$ Main sequence $\cdot$ Monkey

\section{List of symbols}

$\vec{S}=[R, \Phi] \quad$ saccade vector, with amplitude $R$ and direction $\Phi$

$\vec{S}_{0 k}=\left[R_{0 k}, \Phi_{0 k}\right]$ optimal saccade vector for cell $k$ $p(t)$

$\dot{h}, \dot{v}, \dot{p}$

$\ddot{h}, \ddot{v}$

$n_{k}(t)$

$N_{k}$

$N_{0}$

$(u, v)$

$\left(B_{u}, B_{v}\right)$ instantaneous eye displacement along the saccade vector $[0, \mathrm{R}]$ horizontal, vertical and vectorial eye velocity horizontal, vertical eye acceleration instantaneous cumulative number of spikes of cell $k$ total number of spikes in the burst of cell $k$ maximum number of spikes for optimal saccade $( \pm 20)$ collicular anatomical coordinates (in mm re. fovea)

collicular magnification factors along $\mathrm{u}$ and $\mathrm{v}$ axes in $\mathrm{mm}$ and $\mathrm{mm} / \mathrm{rad}$, respectively[$[(1.4,1.8)$ (Ottes et al. 1986); here: $(1.0,1.0)]$

A shift in the SC mapping function in deg [3.0 (Ottes et al. 1986); here: 0]

$\eta, \eta_{d}$ normalization constants of SC population for static (Van Gisbergen et al. 1987), and dynamic model $\left[2.1 \times 10^{-5} \mathrm{deg} / \mathrm{spikes} / \mathrm{s}\right.$ and $3.9 \times 10^{-4} \times \mathrm{deg} /$ spike] 


\begin{tabular}{|c|c|}
\hline $\begin{array}{l}\vec{m}_{k} \\
\vec{s}_{k}\end{array}$ & $\begin{array}{l}\text { movement contribution of cell } k \text { per spike } \\
\text { spike vector of cell } k\end{array}$ \\
\hline$\delta\left(t-t_{k, s}\right)$ & spike kernel of cell $k$ at time $t_{k, s}$ \\
\hline$f_{k}(t)$ & Instantaneous firing rate of cell $\mathrm{k}$ (spikes/s) \\
\hline$F_{0} / F_{k}$ & $\begin{array}{l}\text { peak (or maximum mean) firing rate of the } \\
\text { population/mean firing rate of cell } k\left(F_{0}=\right. \\
800 \text { spikes/s) }\end{array}$ \\
\hline$P$ & number of cells in the active population $( \pm 425)$ \\
\hline oоp & $\begin{array}{l}\text { width of the population in the motor map } \\
(0.5 \mathrm{~mm})\end{array}$ \\
\hline 0 & cell density in SC motor map $\left(86.2 / \mathrm{mm}^{2}\right)$ \\
\hline Dur & time constant of the burst in ms \\
\hline$\sigma_{0}, \beta$ & $\begin{array}{l}\text { time constant of the burst at } R=0 \mathrm{deg} \text {; burst- } \\
\text { duration increment }(3 \mathrm{~ms}, 0.07 \mathrm{~ms} / \mathrm{deg})\end{array}$ \\
\hline$g(t)$ & $\begin{array}{l}\text { normalized activity profile of SC cells gamma } \\
\text { burst }\end{array}$ \\
\hline$\gamma$ & exponent of gamma burst function \\
\hline$e \cdot T_{0}$ & $\begin{array}{l}\text { time-to-peak firing rate of gamma burst }(=\gamma \text {. } \\
\left.\sigma_{\text {Dur }}\right)(30 \mathrm{~ms})\end{array}$ \\
\hline$S$ & $\begin{array}{l}\text { skewness (of eye-velocity profile, or of gamma } \\
\text { burst) }\end{array}$ \\
\hline$V_{\mathrm{pk}}$ & $\begin{array}{l}\text { asymptote of the brainstem nonlinearity } \\
(700 \mathrm{deg} / \mathrm{s})\end{array}$ \\
\hline$M_{0}$ & $\begin{array}{l}\text { angular constant of the burst-generator nonlin- } \\
\text { earity ( } 8 \mathrm{deg})\end{array}$ \\
\hline$B$ & $\begin{array}{l}\text { forward gain of the linear brainstem burst } \\
\text { generator }(80 \mathrm{deg} / \mathrm{s})\end{array}$ \\
\hline & delay in the brainstem feedback loop ( $4 \mathrm{~ms})$ \\
\hline$M_{H, V}(t)$ & $\begin{array}{l}\text { dynamic horizontal/vertical motor error (in } \\
\text { deg) }\end{array}$ \\
\hline$K$ & $\begin{array}{l}\text { offset activity of SC population center-of-grav- } \\
\text { ity ( } 5 \text { spikes/s) }\end{array}$ \\
\hline
\end{tabular}

\section{Introduction}

Saccades are rapid eye movements that redirect the fovea fast and accurately to a peripheral stimulus of interest. In this paper we present a computational model on how a population of neurons in the midbrain superior colliculus (SC) encodes the metrics, kinematics, and trajectories of saccadic eye movements.

First, we will briefly describe the main characteristics of the saccade kinematics: the main-sequence, the shape of the velocity profiles, and straight oblique saccade trajectories (Fig. 1). Next, we will introduce the notion of ensemble coding of saccades by the midbrain SC.

\subsection{Saccadic eye movements: kinematics}

Main-sequence kinematics. The kinematics of visuallyevoked saccades have stereotyped characteristics: the amplitude-duration relation follows a straight line, while peak velocity depends in a nonlinear, saturating way on saccade amplitude. These relations are known as the 'main sequence' of saccades (Bahill et al. 1977). Westheimer (1954) recognized that these kinematic relationships betray a nonlinearity within the saccadic system, as for a linear system movement duration would be fixed for all saccades, and the peak velocity would increase linearly with the saccade amplitude (Fig. 1, left). ${ }^{1}$

The kinematic nonlinearity is typically assigned to a local feedback circuit in the brainstem, in which so-called medium lead burst cells embody the saccadic pulse generator (Luschei and Fuchs 1972; Henn and Cohen 1976; Van Gisbergen et al. 1981). It is thought that these cells are driven by a dynamic motor error signal, which is the difference between the desired endpoint and current eye position. Saccadic burst cells transform this motor error signal into an eye velocity output, and the majority of models of the saccadic system assume that the input-output characteristic of these cells underlies the nonlinear main sequence (Jürgens et al. 1981; Van Gisbergen et al. 1981; Scudder 1988). However, there is actually very little experimental data to support this latter assumption. We will present evidence for an alternative scheme, which proposes that the nonlinearity of the saccade kinematics may be embedded in the spatial-temporal activation patterns of the midbrain SC.

Skewness. A further characteristic property of saccades concerns the shape of their velocity profiles (Fig. 1, center). Typically, the duration of the acceleration phase of saccades (i.e. the time-to-peak velocity) is roughly constant across a wide range of saccade amplitudes. As a result, saccade velocity profiles are skewed. Van Opstal and Van Gisbergen (1989) applied gamma functions to parameterise velocity profiles for different saccade types (visually-evoked, slow saccades due to drugs or fatigue, remembered-target saccades, etc.) and showed that skewness is determined by the saccade duration, rather than by the saccade amplitude.

Oblique saccades. A third kinematic nonlinearity of saccades is 'component stretching' in oblique saccades (Evinger et al. 1981; Van Gisbergen et al. 1985; Smit et al. 1990). Oblique saccades evoked by single visual targets are approximately straight. This simple fact, however, implies that the horizontal and vertical velocity components of the saccade stay scaled versions of each other throughout the movement:

$\dot{v}(t)=c \cdot \dot{h}(t)$

with $c$ a constant. Given the fact that a small saccade has a much shorter duration than a large saccade, Eq. 1 requires a

\footnotetext{
$\overline{1}$ The saturation of peak velocity follows from the straight-line amplitude-duration relationship. For example, if velocity profiles are approximated by triangles, the amplitude-duration relation $D=a+b \cdot R$ yields for the peak velocity relation: $V_{\mathrm{pk}}=2 R /(a+b \cdot R)$, which saturates at $2 / b \mathrm{deg} / \mathrm{s}$.
} 
Fig. 1 Properties of visually-evoked saccades. Left

The main sequence (NL).

Dotted lines (L) indicate responses for a hypothetical linear system. Center Skewness of saccade velocity profiles increases with saccade duration. Right. Component stretching. Here, an oblique saccade in a direction 60 deg re. horizontal $\left(\vec{S}_{60}\right)$ has components with very different amplitudes, but velocity profiles bottom with equal durations and similar shapes. The pure horizontal saccade $\left(\vec{S}_{0}\right)$ has a much shorter duration and higher velocity $\left(\dot{H}_{0}\right)$ than the equally large horizontal component of the oblique saccade $\left(\dot{H}_{60}\right)$
MAIN SEQUENCE
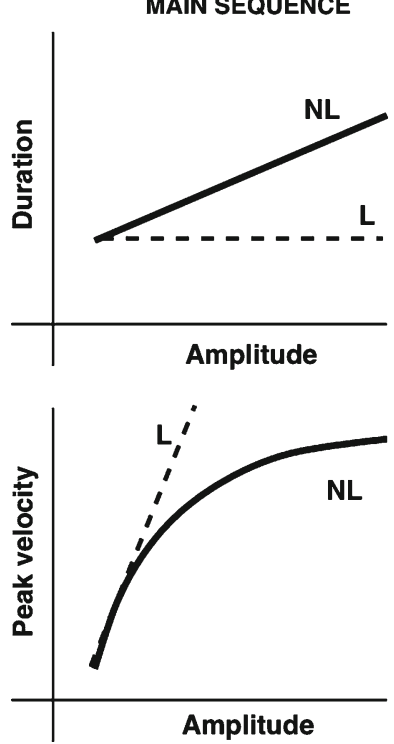
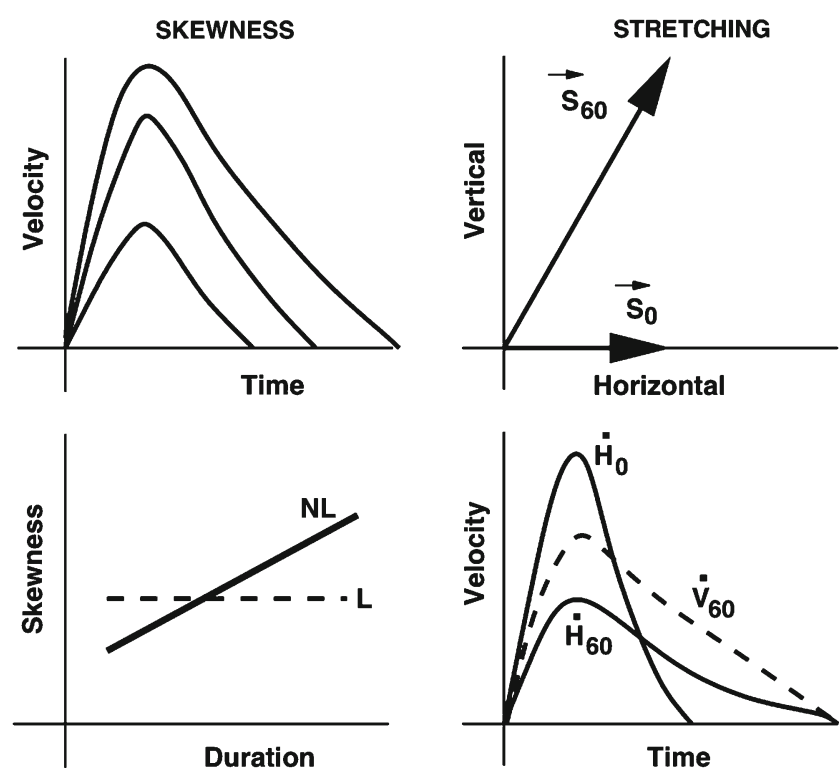

mechanism through which the horizontal and vertical components are coupled, such that the shape of their velocity profiles becomes the same despite large differences in amplitude (Fig. 1, right). Different schemes have been proposed to achieve this coupling. For example, Grossman and Robinson (1988) and Nichols and Sparks (1996) proposed that the horizontal and vertical burst generators have their own independent feedback circuits, but are coupled in such a way that they scale each other's gains. However, when Smit et al. (1990) applied the cross-coupling model to measured oblique saccades they demonstrated that the optimal coupling constants are not fixed, but vary in a complex way with saccade amplitude and direction.

A more obvious way to yield straight saccades results from the common source model (Van Gisbergen et al. 1985; Van Opstal and Van Gisbergen 1989). In this conceptual idea, a nonlinear vectorial pulse generator produces a radial eyevelocity command, $\dot{r}(t)$, from which the horizontal and vertical velocity components are subsequently derived by linear vector decomposition:

$\dot{h}(t)=\cos (\Phi) \cdot \dot{r}(t) \quad$ and $\quad \dot{v}(t)=\sin (\Phi) \cdot \dot{r}(t)$

Note that from Eq. $1 c=\tan (\Phi)$, and that in the commonsource scheme component stretching and straight saccades are an emerging property of a shared nonlinear vectorial pulse generator. In cross-coupling models, however, stretching is an additional nonlinear design property needed to account for straight saccades. Although the common-source scheme fitted measured oblique saccade data better than the crosscoupled scheme (Smit et al. 1990), it assumed a vectorial pulse generator for which, at the time, no neurophysiological evidence was available. Here we will argue that the midbrain SC could serve this function.

\subsection{Superior colliculus}

The SC is crucial for the generation of saccades. Its deeper layers contain a topographic map of saccade vectors, which is organized in oculocentric coordinates, as electrical microstimulation produces fixed-vector saccades, the amplitude and direction of which are determined by the site of stimulation within the map (Robinson 1972; Klier et al. 2001). The stimulation parameters have a systematic effect on the saccade properties: low-frequency stimulation produces slower saccades than high-frequency stimulation (Stanford and Sparks 1996), whereas at low current strengths the evoked saccade amplitude is reduced (Van Opstal and Van Gisbergen 1990b).

Cells in the motor map fire a brisk burst of action potentials tightly coupled to the onset and duration of the saccade. Near the rostral pole of the SC cells are involved in the generation of small saccades, while at caudal sites cells encode large eye movements. The range of movement vectors for which an SC neuron is recruited is called its movement field (Sparks et al. 1976; Ottes et al. 1986).

Figure 2a-c shows a typical example of a SC movement field for a cell with an optimal saccade vector of $\vec{S}_{0}=$ $[13,230] \mathrm{deg}$, together with the average saccadic eye movement evoked by micro stimulation at the recording site. Note that the evoked saccade (black traces) is almost indistinguishable from the optimal visually-evoked response (gray traces). In Fig. $2 d$ it can be seen that electrically evoked saccades closely correspond with the optimal saccades encoded by cells near the stimulation electrode.

It is generally assumed that the location of the active population of cells in the motor map carries information about the amplitude $(R)$ and direction $(\Phi)$ of the upcoming saccade vector. There exists strong support for the notion that activity in the SC motor map is also related to, and may 


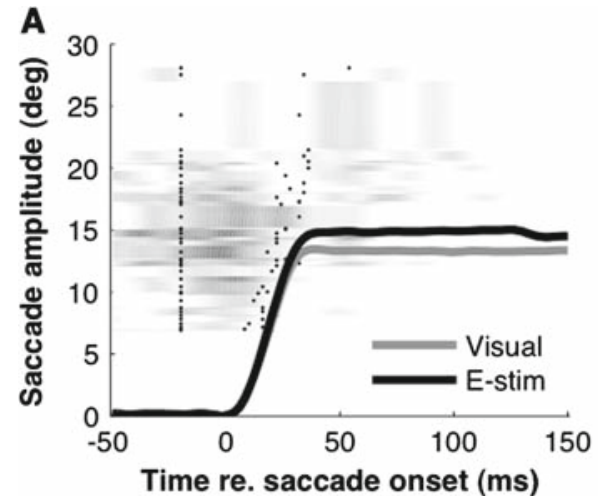

B

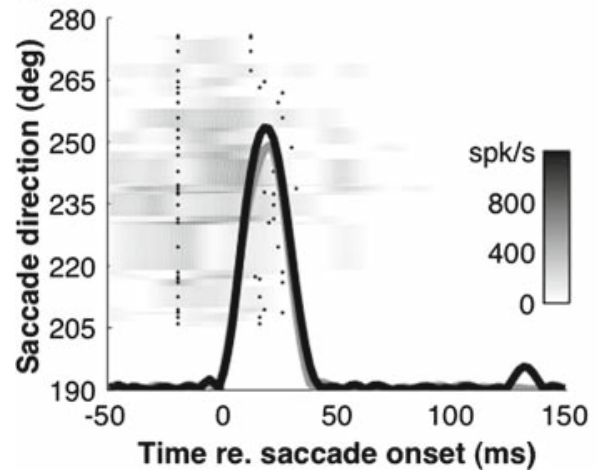

Fig. 2 Movement field of superior colliculus neuron pj9003. a Instantaneous firing rate (gray scale) as function of time, for saccades through the center of its movement field, sorted for different amplitudes (amplitude scan). Tick marks indicate spike-counting windows for saccaderelated burst ( $20 \mathrm{~ms}$ before saccade onset to $20 \mathrm{~ms}$ before offset). Gray trace is the average eye position for the cell's optimal visually-evoked saccade. Black trace corresponds to the average saccade elicited by micro-stimulation at the recording site. Note close correspondence. b Same for a direction scan through the movement field center. Velocity

even be responsible for, the kinematic properties of saccades (Berthoz et al. 1986; Van Opstal and Van Gisbergen 1990b; Waitzman et al. 1991; Munoz et al. 1996; Stanford et al. 1996; Soetedjo et al. 2002; Matsuo et al. 2004). The precise nature of the collicular involvement in the dynamic control of saccades has been controversial, and will be the main topic of this paper.

We will expand on our recent finding that the instantaneous activity of saccade-related cells in the SC faithfully reflects the instantaneous displacement of the eye in the direction of the planned saccade vector (Goossens and Van Opstal 2006). This hypothesis was based on the results of an experimental paradigm in which monkeys made saccades under open-loop conditions toward a briefly flashed visual target. A representative example of this paradigm is shown in Fig. 3 . In some trials, an unexpected brief air puff evoked a blink response that coincided with the saccadic eye movement. In those trials, the saccade trajectories were heavily perturbed, and highly variable. Moreover, peak eye velocities decreased
C
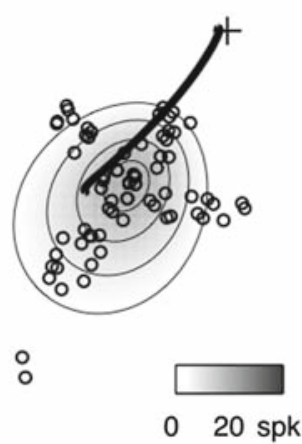

D

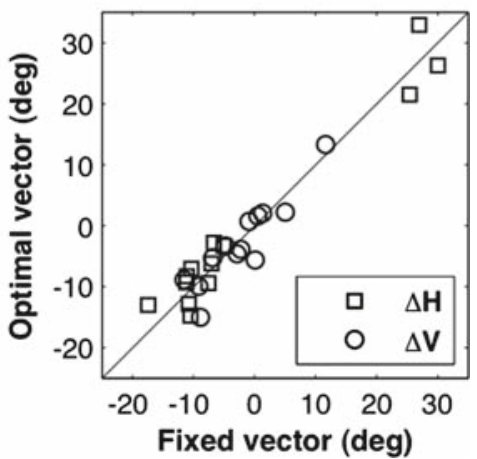

profiles of the saccades are superimposed. c Spatial extent of the movement field. Gray scale is number of spikes in the burst. Contour lines drawn at $[0.5,1.0,1.5$ and 2$] \cdot \sigma_{\text {pop }}$. Circles denote saccade end points shown in the left panels. Black trace is the average stimulation-induced saccade trajectory, which ends closely to the center of the movement field. d Close correspondence between the optimal saccade vectors of 13 different cells and the fixed-vector stimulation-induced saccades at the recording sites

by $40 \%$ or more, and saccade durations increased, often twoto threefold (Fig. 3a, center). Despite these strong perturbations, the saccade endpoint accuracy was virtually unaffected (Fig. 3b, inset). In other words, the saccade displacement vector remained the same (Goossens and Van Opstal 2000a).

Also the saccade-related activity in the SC was strongly affected by the blink perturbations: the mean- and peak-firing rate in the burst dropped substantially (Fig. 3a, top), while the burst duration increased, matching the longer saccade duration (Goossens and Van Opstal 2000b). However, the relation between instantaneous firing rate and dynamic motor error, which is nearly linear in control saccades (Waitzman et al. 1990), broke down in perturbed trials (Goossens and Van Opstal 2000b; Keller and Edelman 1994; Munoz et al. 1996). This indicated that the SC cells do not encode the instantaneous motor error.

Interestingly, the number of spikes in the saccade-related burst was the same for perturbed and control saccades (Fig. 3a, bottom). A similar observation had been made earlier 


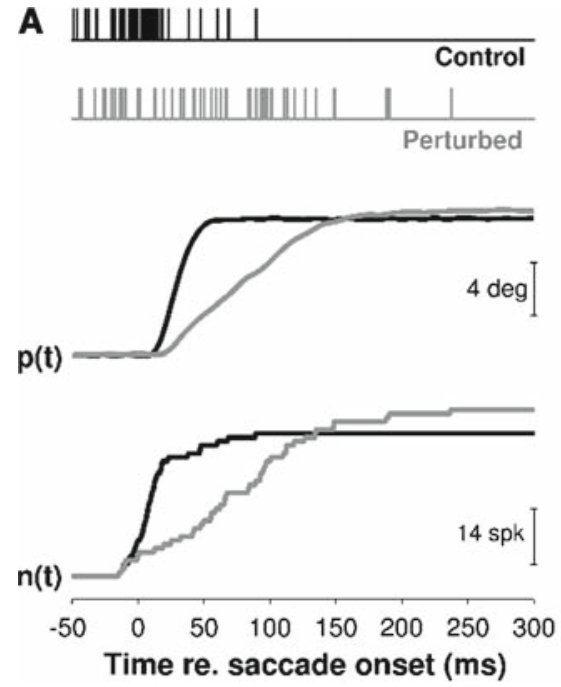

Fig. 3 Responses of SC neuron er0902 for 10 deg saccades illustrating the results obtained with the blink perturbation paradigm. a Discharge pattern during a typical control and perturbation trial together with corresponding traces of eye displacement, $\mathrm{p}(\mathrm{t})$, and cumulative number of spikes, $\mathrm{n}(\mathrm{t})$, as a function of time. $\mathrm{p}(\mathrm{t})$ is the instantaneous eye-displacement component in the direction of the saccade vector $\vec{S}=[R, \Phi]$, given by: $p(t)=h(t) \cdot \cos (\Phi)-v(t) \cdot \sin (\Phi)$. b Delayed $(20 \mathrm{~ms})$ cumulative spike counts as function of eye displacement, $\mathrm{p}(\mathrm{t})$. Dots are data from additional control and perturbation trials. Inset: 2D saccade trajectories of (a) superimposed on the cell's movement field.

by Munoz et al. (1996) for saccades that were briefly interrupted by stimulation in the brainstem omnipause region.

Thus, our data show a clear link between the detailed SC spike trains and the ensuing eye-movement, but not that such is determined by dynamic feedback at the level of the SC. Instead, the data show that the short-latency changes in SC firing observed during blinking are caused by trigeminal reflex mechanisms, and that these changes precede changes in eye velocity by about $20 \mathrm{~ms}$. In addition, we found that that the changes in firing rates were unrelated to the blink-induced curvature in the eye-movement trajectories (Goossens and Van Opstal, 200b; see Fig. 3). Taken together, we believe that our results strongly promote the idea that the SC motor map produces an eye-movement command that is generated upstream from the local feedback loop that controls the saccade burst generator. Similar suggestions have been made by Nichols and Sparks (1996), Crawford and Guitton (1997), and Kato et al. (2006).

Our findings led to the hypothesis (Goossens and Van Opstal 2006) that the instantaneous cumulative number of spikes in the burst, $n(t)$, reflects the instantaneous eye displacement along the saccade vector, $p(t)$. The data indeed showed that the saccade-related burst of all SC cells could be well described by this idea, both for control and for highly perturbed saccades (Fig. 3b).

At first glance, this hypothesis may seem to suggest that the motor output of the SC would merely encode a directional

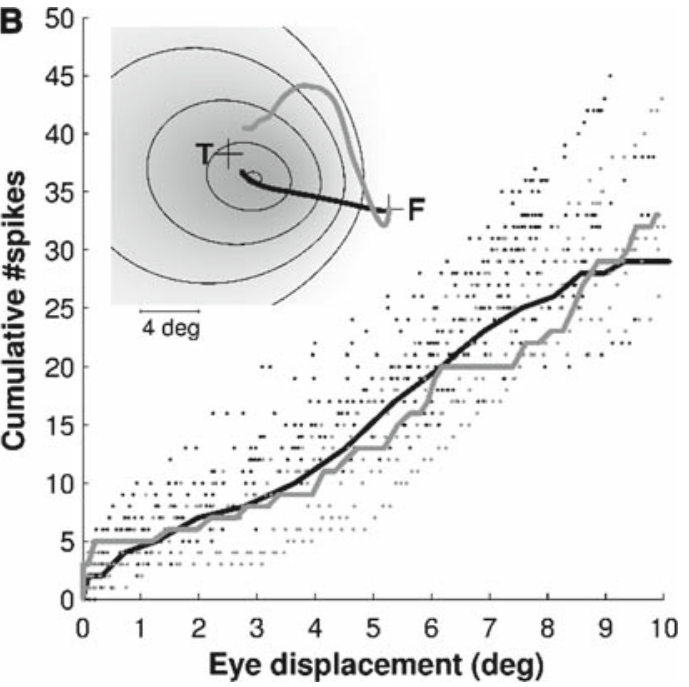

Note robust changes in eye velocity, saccade duration and 2D trajectories as well as in mean and peak firing rates and burst duration. Only saccade accuracy and numbers of spikes in burst remained unaffected. Also note that the response curves for control and perturbed saccades in (b) follow very similar and roughly linear trajectories. Thus the firing patterns of this cell reflected neither the curvature of the eye movement nor the subsequent compensatory phase, but were faithfully related to the intended straight (1D) eye displacement trajectory. Comparable results were obtained for all 25 neurons that could be fully tested with this paradigm (Goossens and Van Opstal 2006)

signal for the saccade (as proposed by Quaia et al. 1999, and more recently also by Arai and Keller 2005). Instead, however, our hypothesis entails that the motor SC emits a dynamic vectorial velocity command that faithfully reflects the actual saccade trajectory and kinematics. For example, our model predicts curved saccade trajectories when additional and delayed activity would arise in the motor map at another location (which may happen when saccades are elicited by multiple stimuli, e.g. Port and Wurtz 2003; see also Arai and Keller 2005). However, trajectory perturbations imposed at a level downstream from the SC (like in blinking) are not observed in the spatial-temporal SC firing patterns, due to the lack of a feedback signal to the motor map.

The consequence of our simple formulation is (1) that each spike in the SC contributes a fixed, tiny movement vector to the saccade, and (2) that movement fields of SC cells are dynamic. These predictions were fully explored in Goossens and Van Opstal (2006) and led to the formulation of the dynamic linear ensemble-coding model, which will be presented in detail below (Fig. 4). Before introducing this model, we will first briefly review the concept of ensemble coding by the SC.

\subsection{Ensemble coding: static}

The idea of ensemble coding proposes that a large population of coarsely-tuned neurons in the SC motor map is 

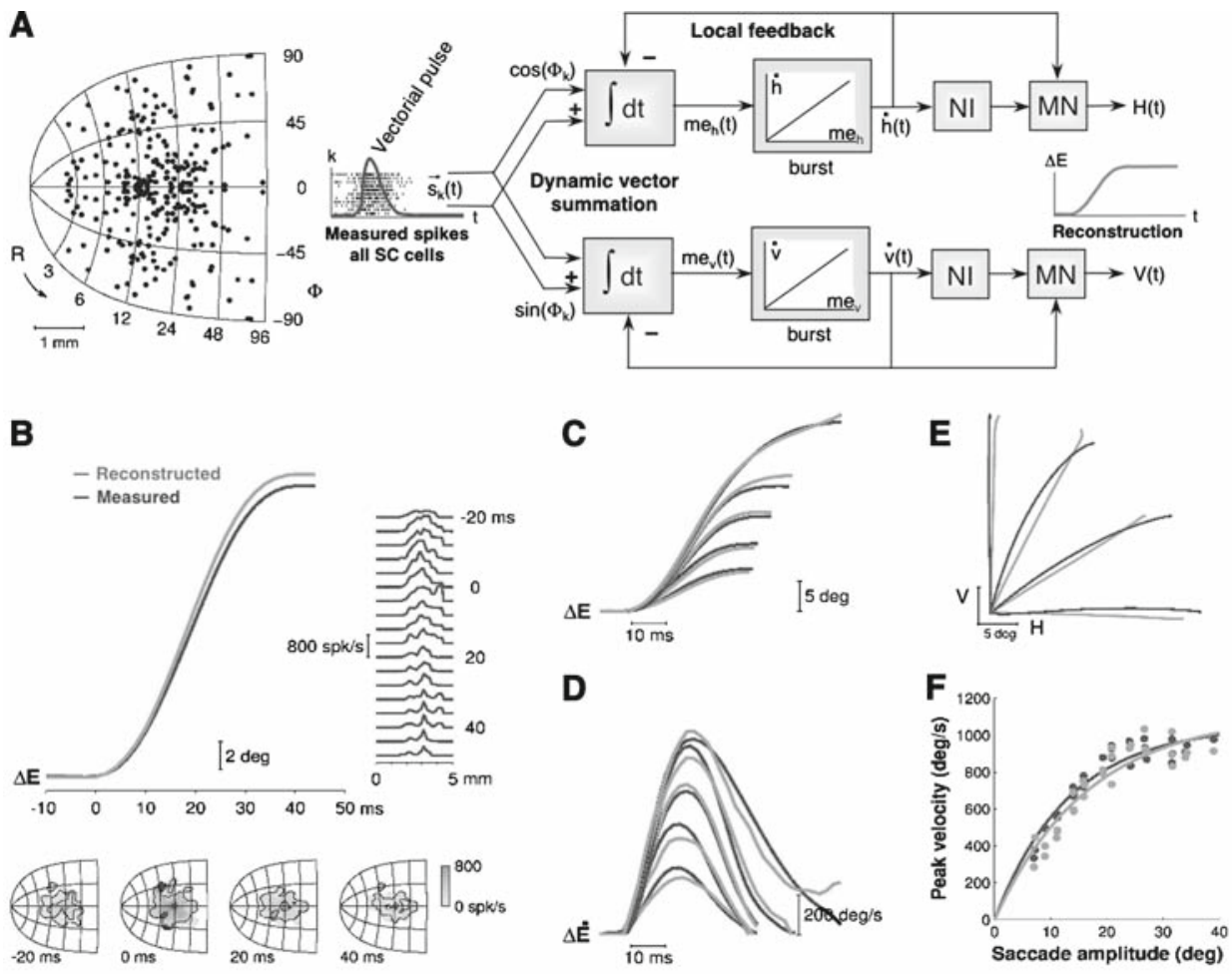

Fig. 4 Reconstruction of saccades from measured SC activity patterns.a Linear 2D model of the SC brainstem saccade generator. In this model, each spike from each cell $\mathrm{k}$ contributes a tiny "spike vector", $\vec{s}_{k}(t)$, to the eye displacement command. The instantaneous sum of spike vectors from all cells in the active population thus represents a vectorial velocity pulse. Dots in the SC map indicate locations of recorded and mirror-reflected cells. NI, neural integrator; MN, motor neurons. b Raw discharge patterns of all SC cells for a rightward saccade of $\sim 20 \mathrm{deg}$ applied to the model. The reconstruction produced a realistic saccadic profile that closely matched the (average) measured saccade. Insets show maps of instantaneous firing rates in the contralateral SC (bottom) and distribution of activity along its rostral-to-caudal axis (left; $0 \mathrm{~mm}$ corresponds to rostral pole) at different moments in time. c,d Reconstructions generated realistic eye displacement and velocity profiles for saccades of different amplitudes $\left(R \in[7,11,16,21,32]^{\circ} ; \Phi=30^{\circ}\right)$. eThe horizontal and vertical saccade components show the correct amount of "stretching" needed to obtain straight saccades in all directions, even though the scheme does not assume the planning of a straight saccade. It is a "multiple source" model with independent horizontal and vertical burst generators. f Reconstructed saccades showed the same nonlinear, saturating amplitude-peak velocity relation ("main sequence") as the measured saccades, even though the brainstem circuit in the model is entirely linear

Here, the complex-logarithmic afferent mapping function $[R, \Phi] \rightarrow[u, v]$ is given by:

$u(R, \Phi)=B_{u} \ln \left(\frac{\sqrt{R^{2}+2 A R \cos \Phi+A^{2}}}{A}\right)$ and

$v(R, \Phi)=B_{v} \arctan \left(\frac{R \sin \Phi}{R \cos \Phi+A}\right)$

The mapping parameters $\left[B_{u}, B_{v}, A\right]=[1.4 \mathrm{~mm}, 1.8 \mathrm{~mm} / \mathrm{rad}$, $3.0 \mathrm{deg}$ ] were determined from Robinson (1972) microstimulation data. The model describes the mean firing rate of a given cell in the SC motor map for any saccade vector with amplitude $R$ and direction $\Phi$, and accounts well for the observed asymmetric shape of SC movement fields (e.g. Fig. 2a,b). It also accounts for the fact that cells encoding small saccades have movement fields extending over a much smaller amplitude range than cells recruited for large saccades. 
Van Gisbergen et al. (1987) applied this model in an attempt to understand how the neural population of Eq. 3 could then encode the planned saccade vector. To that end, they assumed that each recruited cell, $k$, located in the motor map at $\left[u_{k}, v_{k}\right]$ would contribute a tiny movement vector, $\vec{m}_{k}$, with a size, $r_{k}$, and direction, $\phi_{k}$, that was fully determined by its fixed efferent projections to the horizontal $\left(x_{k}\right)$ and vertical $\left(y_{k}\right)$ brainstem burst generators, according to:

$x_{k}=\eta \cdot A \cdot \exp \left(\frac{u_{k}}{B_{u}}\right) \cdot\left[\cos \left(\frac{v_{k}}{B_{v}}\right)-1\right]$ and

$y_{k}=\eta \cdot A \cdot \exp \left(\frac{u_{k}}{B_{u}}\right) \cdot \sin \left(\frac{v_{k}}{B_{v}}\right)$

Note that the efferent mapping of Eq. $5,[u, v] \rightarrow[R, \Phi]$ is the inverse of the afferent mapping of Eq. 4 (apart from the fixed scaling $\eta=1 /\left(F_{0} \cdot \sigma_{\text {pop }}^{2} \cdot 2 \pi \cdot \rho_{0}\right)$, with $\rho_{0}$ the number of cells per $\mathrm{mm}^{2}$ ). A cell's preferred movement vector is hence given by $\vec{S}_{0 k}=\vec{m}_{k} / \eta$.

In the ensemble-coding model of Van Gisbergen et al. (1987) the saccade vector, $\vec{S}=[R, \Phi]$, is then determined by linear summation of all cell contributions in the population, multiplied by their mean firing rates, $F_{k}$ :

$\vec{S}=\sum_{k=1}^{P} F_{k} \cdot \vec{m}_{k}$

with $P$ the total number of cells in the population. The model of Van Gisbergen et al. (1987) explains how a large, invariant population (Eq. 3) within a logarithmically compressed motor map (Eq. 4) can represent saccade vectors with the correct amplitude and direction throughout the oculomotor range, and accounts for the shape of SC movement fields without any further adjustment of parameters (Fig. 2).

However, the model did not incorporate a mechanism to explain the saccade kinematics, nor did it account for component stretching in oblique saccades. Thus, it remained unclear how to combine the ensemble-coding scheme of Eq. 6 with either the common source model of Eq. 2, or with mutually coupled brainstem burst generators, although Smit et al. (1990) and Van Gisbergen and Van Opstal (1989) speculated that perhaps the vectorial pulse generator was embodied by the motor SC.

Lee et al. (1988) noted a further problem with the linear summation model. They argued, on the basis of local reversible inactivation experiments within the motor map, that vector averaging could better account for the observed data than linear summation. Thus, according to their proposal, the saccade vector was computed by:

$\vec{S}=\sum_{k=1}^{P} F_{k} \cdot \vec{S}_{0 k} / \sum_{k=1}^{P} F_{k}$
Note that Eq. 7 incorporates normalization by the total activity within the population. This requires a nonlinear mechanism for which it is not obvious how it might be implemented through fixed SC-brainstem connections.

\subsection{Ensemble coding model: dynamic}

The ensemble-coding model of Goossens and Van Opstal (2006) was proposed to capture the findings of the blinkperturbation paradigm (see above, Fig. 3). In contrast to the static ensemble-coding model (Eq. 6) of Van Gisbergen et al. (1987), they introduced dynamics into the motor map output, by assuming that each spike $s$ of each cell $k$ in the motor map, fired at time $t_{s, k}$ generates a tiny movement vector, here called a 'spike vector', which is given by:

$\vec{s}_{k}(t)=\vec{m}_{k} \cdot \delta\left(t-t_{s, k}\right)$

Here, $\vec{m}_{k}$ is the effective connection vector of SC neuron $k$ to the brainstem burst generator (Eq. 5), and the spike kernel $\delta\left(t-t_{s, k}\right)=1$ for $t=t_{s, k}$ and 0 elsewhere.

Furthermore, $\vec{S}_{0 k}=\vec{m}_{k} / \eta_{d}$ is the cell's preferred movement vector (i.e. the center of its movement field), where the population scaling factor is determined by $\eta_{d}=1 /\left(N_{0} \cdot \sigma_{\text {pop }}^{2}\right.$. $\left.2 \pi \cdot \rho_{0}\right)$. Here, $N_{0}$ is the fixed maximum number of spikes in the population.

The planned saccade trajectory, generated by the population of recruited neurons, is then determined by instantaneous linear summation (i.e. temporal integration) of all spike vectors:

$\vec{S}(t)=\sum_{k=1}^{P} \sum_{s=1}^{N_{k}} \vec{s}_{k}(t)$

with $P$ the total number of cells in the population, and $N_{k}$ the total number of spikes (fired at times $t_{s, k}$ ) in the burst of cell $k$. The model has a similar structure as Scudder (1988) local feedback model, and is presented in Fig. 4a. Figure 4b-f shows a number of measured (dark grey) saccade trajectories, with the corresponding simulated (light grey) saccades, based on the recorded spikes from the neural population.

Several predictions of this simple model on the behaviour of SC cells were verified in Goossens and Van Opstal (2006), for which the most important ones are:

(i) Movement fields of SC cells are dynamic: for saccades of any amplitude and direction the cumulative number of spikes in the burst of cell $k, n_{k}(t)$, predicts how far the eye has moved along either the cell's preferred movement vector, $\vec{S}_{0 k}=\left[R_{0 k}, \Phi_{0 k}\right]$, or along the actual saccade vector, $\vec{S}_{=}[R, \Phi]$, i.e.:

$n_{k}(t)=a_{k} \cdot p(t)$ 
with $a_{k}$ the slope in \#spikes/deg, and $p(t) \in[0, R]$ the instantaneous eye displacement along the saccade vector $\vec{S}$ (Fig. 3b).

(ii) The slope of Eq. 10 is determined by $a_{k}=N_{k}(R, \Phi) / R$, in which $N_{k}(R, \Phi)$ (the total number of spikes in the burst of cell $k$ for the saccade) replaces the mean firing rate $F_{k}$ in the static movement field description of Eq. 3.

(iii) Equation 10 holds for fast, as well as for extremely slow (e.g. blink-perturbed) saccades to the same target.

(iv) With the additional assumption that the total number of spikes of the saccade population is fixed for all saccade amplitudes, and that this number controls the saccade offset (e.g. through a downstream threshold mechanism), the results of the micro-lesion experiments of Lee et al. (1988) could be explained without the need for the nonlinear vector averaging scheme of Eq. 7 (Goossens and Van Opstal 2006).

To test the possibility that the SC population encoded the saccade kinematics, the result of Eq. 9 was fed into two independent, but linear, feedback circuits that represented the horizontal and vertical saccade burst generators.

When this simple model was applied to actual recordings taken from 140 saccade-related burst neurons during thousands of saccades, it was able to reproduce the metrics, straight trajectories, the main-sequence kinematics and velocity profiles of saccades to single visual targets across the oculomotor range (Goossens and Van Opstal 2006, see Fig. 4). Note that in the reconstruction of these saccades the neuronal activity was not normalised in any way. Quite remarkably, only three free parameters (the forward gains, $B_{H}$ and $B_{V}$, and the delay, $\tau$, in the brainstem feedback loops) sufficed to produce excellent correspondence between real saccades and model saccades.

Because the applied SC-brainstem model is entirely linear, the fact that reconstructed saccades were straight and obeyed the nonlinear main sequence had to result from the properties of the model's input, i.e. the measured spatial-temporal activity patterns in the SC motor map. In other words, the saccade reconstructions strongly suggested that the motor SC might act as a nonlinear vectorial pulse generator.

The question as to which aspects of the input patterns could be responsible for these properties was not explored in the Goossens and Van Opstal (2006) study. Therefore, the present paper proposes a mechanism through which the SC population could generate the nonlinear saccade kinematics.

\section{Methods}

Simulations were performed in Matlab 7.4 (version R2007a) with a simplified description of the afferent collicular motor map:
$u(R)=B_{u} \cdot \ln (R) \quad$ and $\quad v(\Phi)=B_{v} \cdot \Phi$

with $B_{u}=1 \mathrm{~mm}$ and $B_{v}=1 \mathrm{~mm} / \mathrm{rad}$ (isotropic map). This yields for the efferent mapping function (cf. Eq. 5):

$x=\exp (u) \cdot \cos (v)$ and $y=\exp (u) \cdot \sin (v)$

The instantaneous firing rate of cell $k$ (at location $\left(u_{k}, v_{k}\right)$ in the motor map) during a saccade with coordinates $[R, \Phi]$ (with point image $\left[u_{R}, v_{\Phi}\right]$ in the motor map) was described by:

$f_{k}(t)=F_{0} \exp \left(-\frac{\left(u_{k}-u_{R}\right)^{2}+\left(v_{k}-v_{\Phi}\right)^{2}}{2 \sigma_{\text {pop }}^{2}}\right) \cdot g(t)$

with $g(t)$ the cell's temporal activity profile. In our simulations, we applied a normalised gamma burst to describe this temporal behaviour:

$$
\begin{aligned}
g(t)= & T_{0}^{\gamma} \cdot\left(t-t_{\mathrm{ON}}\right)^{\gamma} \cdot \exp \left(-\frac{\left(t-t_{\mathrm{ON}}\right)}{\sigma_{\mathrm{DUR}}}\right) \\
& \text { with } T_{0}=\sigma_{\mathrm{DUR}} \gamma / e \text { and } t \geq t_{\mathrm{ON}}
\end{aligned}
$$

Note that the amplitude of Eq. 14 is normalised to one, $t_{\mathrm{ON}}$ is the burst onset, and $\sigma_{\text {Dur }}$ is a measure for the burst duration. The exponent $\gamma$ determines the gamma-burst skewness by $S=2 / \sqrt{(\gamma+1)}$, and $T_{0} \cdot e=\sigma_{\text {Dur }} \cdot \gamma$ is the time-to-peak firing in the gamma burst.

The motor map consisted of a rectangular $[u, v]$ grid of $51 \times 51$ neurons, in which $-4.8 \leq u \leq+4.8 \mathrm{~mm}$ and $-\pi / 2 \leq v \leq+\pi / 2 \mathrm{~mm}$. We took a fixed population width of $\sigma_{\text {pop }}=0.5 \mathrm{~mm}$, and the scaling constant $\eta_{d}=3.9 \times$ $10^{-4} \mathrm{deg} /$ spike was determined by tuning the model for a $20 \mathrm{deg}$ horizontal saccade. For each saccade a population of about $P=425$ cells was recruited, which generated a fixed number of about 2,540 spikes. The central cell in the population fired between 19-20 spikes.

In our simulations the linear brainstem was given identical gains of $B_{H}=B_{V}=80 \mathrm{deg} / \mathrm{s}$, with a feedback delay of $\tau=4 \mathrm{~ms}$, for both the horizontal and vertical eye-movement components (see Fig. 4). For simplicity, the plant transfer characteristics were ignored. Thus, the model's saccade trajectories were completely reflected in the SC population output of Eq. 9.

Figure 5 shows a movement field scan for a model neuron with an optimal saccade vector of $[R, \Phi]=[15,0] \mathrm{deg}$. Figure 5a shows a classical static movement field description in which the cell's activity is quantified by the total number of spikes in the burst for saccades in the optimal (horizontal) direction (left), and for optimal saccade amplitudes (15 deg) in different directions (right). Fig $5 \mathrm{~b}$ shows the gamma-burst activity for this unit as function of time (Eq. $15 ; T_{0}=11 \mathrm{~ms}$ ) for the same saccade vectors as in Fig. 5a. Note that these model responses correspond well with the actual data shown in Fig. 2. 

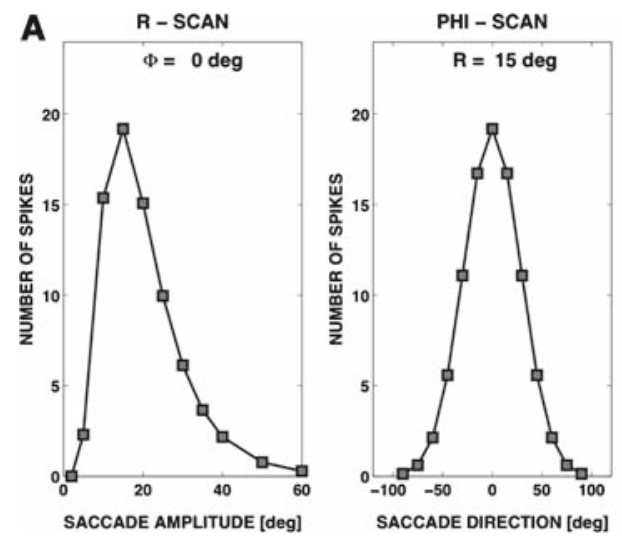

Fig. 5 Movement field of a SC model cell with an optimal saccade vector of $[R, \Phi]=[15,0]$ deg. a Left: Total number of spikes in the gamma-burst as function of saccade amplitude (direction $0 \mathrm{deg}$ ); right: number of spikes as function of saccade direction (amplitude $15 \mathrm{deg}$ ). As

\section{Results}

As mentioned in Sect. 2, despite the logarithmic compression of the afferent mapping function (Eq. 4), the saccadic output of the total population has linear kinematics when the parameters describing the cell activity in Eqs. $13-14, F_{\mathrm{pk}}, \sigma_{\mathrm{pop}}$ and $\sigma_{\text {Dur }}$, are independent of the saccade amplitude, or of the cell's location in the motor map. This property of the model is illustrated in Fig. 6, which shows a number of horizontal saccadic eye movements (Fig. 6a) with their corresponding velocity profiles (Fig. 6b), together with the resulting mainsequence relations (inset Fig. 6b).

The reason for this overall linear behaviour is that the exponential nature of the efferent mapping function exactly cancels the logarithmic compression of the afferent map, and that this apparently also holds when a large population of cells is involved. Thus, if the population activity profile is taken invariant across the motor map (i.e. fixed values for $F_{0}, \sigma_{\text {pop }}$ and $\sigma_{\text {Dur }}$ for all cells in the SC), the ensemble-coding model of Eq. 9 will not yield a nonlinear main sequence. Instead, saccade durations will be constant for all amplitudes, and the
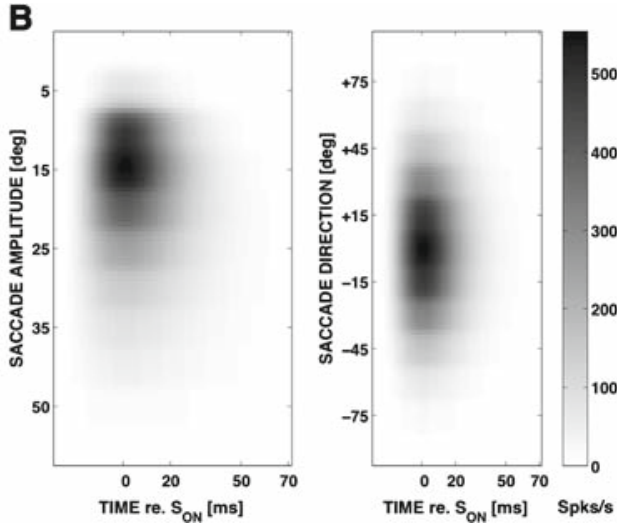

a result of the logarithmic compression of the motor map, the amplitude tuning curve is asymmetric, in contrast to the direction tuning curve. b Gamma-burst activity profiles for the amplitude (left) and direction (right) scans. Scale: firing rate in spikes/s

peak eye velocity will vary linearly with saccade amplitude (Fig. 6b, inset). Also the skewness of the saccade velocity profiles will be fixed (cf. Fig. 1).

Note that a fixed population size, $\sigma_{\text {pop }}=0.5 \mathrm{~mm}$, together with a homogeneous cell density across the SC, implies that the total number of cells contributing to any visual evoked saccade is constant. And if the firing rates $F_{\mathrm{pk}}$ and burst durations $\sigma_{\text {Dur }}$ are the same for each location in the motor map, the total number of spikes emitted by the population is the same too. Experimental evidence given in Goossens and Van Opstal (2006, their Fig. 7) indeed support the assumption that the size of the recruited population as well as the total number of spikes emitted by the population are the same for each saccade vector. The question therefore arises, whether the other burst properties are independent of a cell's location in the motor map.

In Fig. 7a (top, left) we show that the number of spikes of cells in the motor SC for their optimal saccades is the same across the motor map, as the correlation between the spike count and optimal saccade amplitude is not significant. The bottom panel in Fig. 7a, however, shows an additional
Fig. 6 The model behaves as a linear system, when the temporal burst profiles have fixed parameters. a Eye position traces. b Velocity profiles scale linearly with saccade amplitude. Inset: main sequence
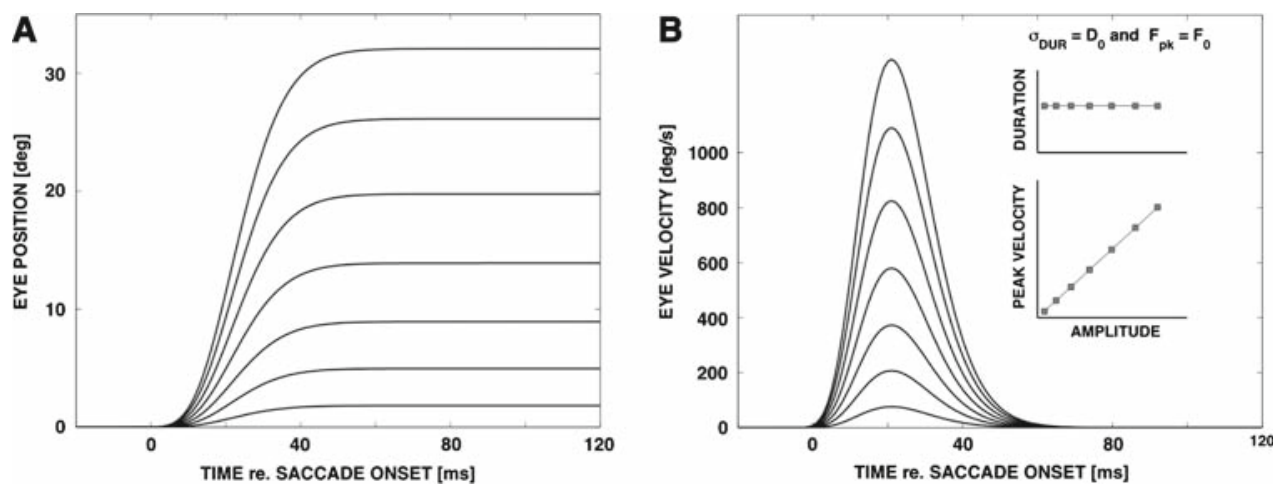

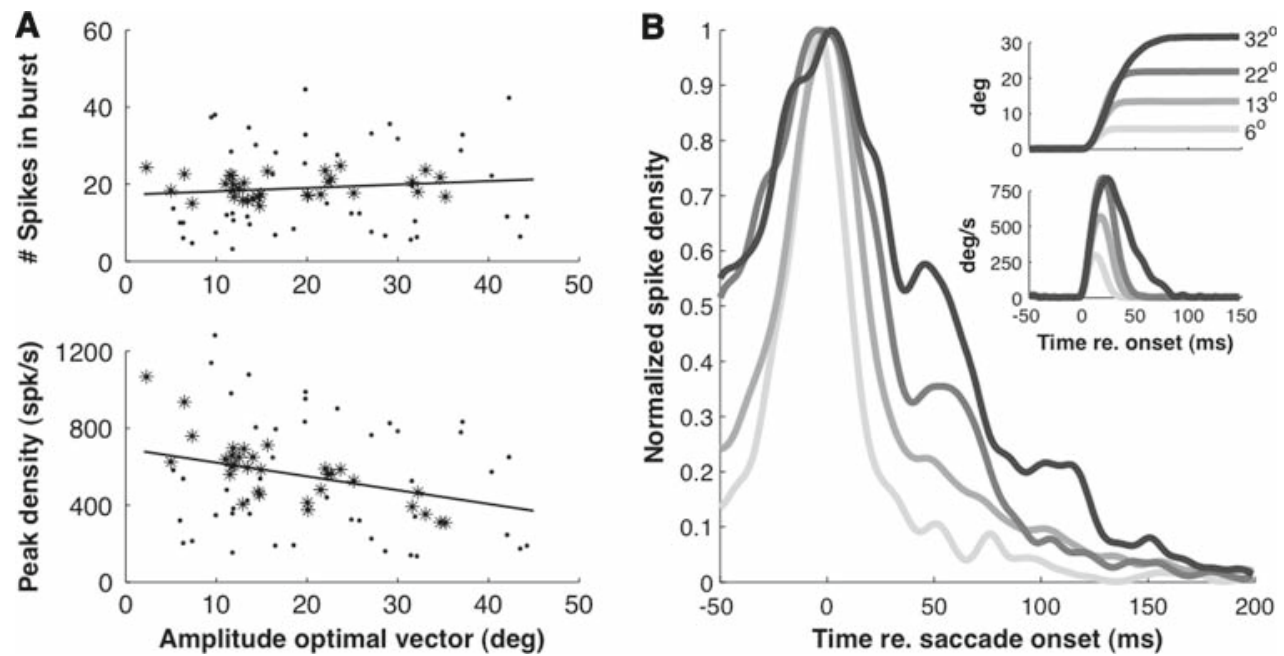

Fig. 7 Burst properties of SC saccade-related cells. Cells (N=77) were selected for having at least five saccades into the center of their movement field (within $\left.0.5 \sigma_{\text {pop }}\right)$. Highlighted cells $(\mathrm{n}=32)$ are selected for producing $N_{0}=20$ spikes for their optimal saccade. a Top: Number of spikes is not related to the optimal saccade amplitude. Bottom: Peak firing rate of the spike-density function, however, decreases systemati-

cally as function of a cell's optimal amplitude b Average spike-density burst profiles (peaks normalised) for the four clusters of cells show a clear increase of burst duration (and skewness) with saccade amplitude. Inset: average optimal radial saccade position—and velocity traces for the four cell groups. Note main-sequence behaviour and skewness
Fig. 8 a Peak firing rate, burst duration (width, $\sigma_{\text {Dur }}$; left axis), the number of spikes, and burst skewness (right axis) of each model cell as function of its optimal saccade amplitude (Eqs. 14-15). b Examples of SC gamma bursts with the properties shown in a
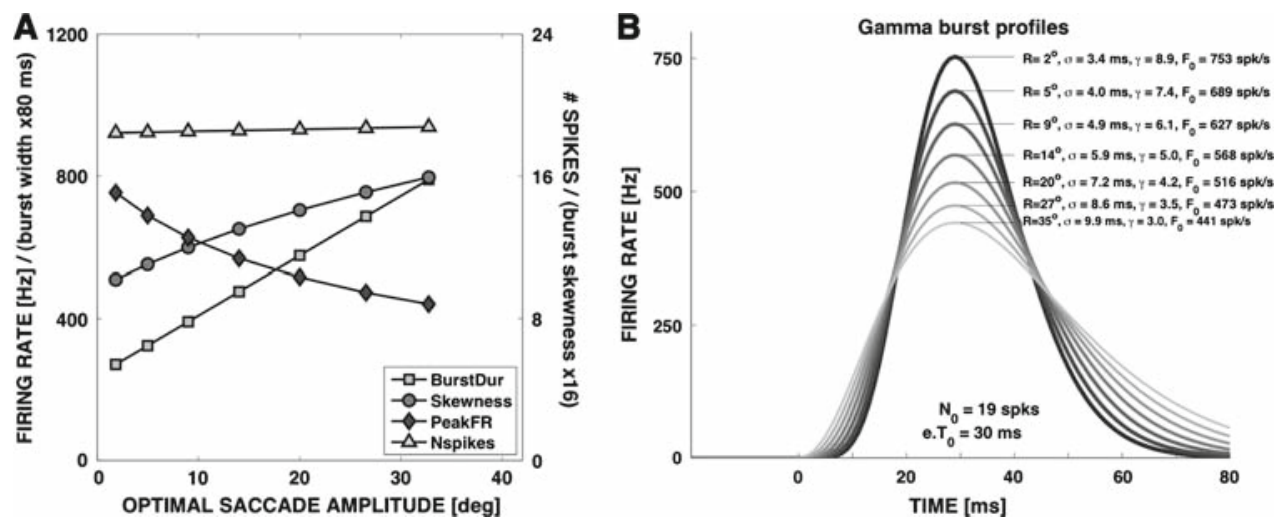

analysis for these cells, which indicates that the peak firing rate of SC cells varies systematically with their optimal saccade amplitude.

Figure $8 \mathrm{~b}$ shows the normalised temporal burst profiles for the four selected amplitude clusters for which $N_{0}=20$ spikes (32 cells, asterisks in Fig. 7a). Note that the peak-firing rate occurs at about the same instant re. saccade onset, while the burst duration clearly increases with the optimal amplitude. Hence, also the burst skewness increases with saccade amplitude. The gamma function of Eq. 14 therefore seems to be a reasonable description for the shape of the saccaderelated bursts.

Thus, in contrast to the simplified assumption made in the simulations of Fig. 6, the burst characteristics of SC cells $d o$ appear to vary in a systematic way with their optimal saccade vector (i.e. with their location in the motor map). To investigate whether the nonlinear main sequence properties may result from neural populations endowed with these characteristics, we incorporated these features into the spike-vector model.

To that end, we let the burst duration as well as a cell's peak firing rate depend on the saccade amplitude, $R$, according to: ${ }^{2}$

$\sigma_{\text {Dur }}(R)=\sigma_{0} \cdot[1+\beta \cdot R]$ and $F_{\mathrm{pk}}(R)=\frac{F_{0}}{\sqrt{1+\beta \cdot R}}$

in which $F_{0}, \sigma_{0}$ and $\beta$ are constants (see Fig. 8a). In accordance with the data shown in Fig. 8, cells near the rostral pole

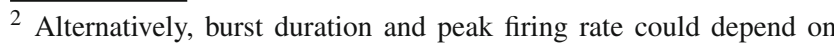
the cell's optimal saccade amplitude, $R_{0 k}$. In that case, the burst properties depend exclusively on the cell's location in the motor map, as $R_{0 k}=\exp \left(u_{k}\right)$, rather than on the actual saccade, $R$ (Eq. 10). See also Sect. 4 for this subtle, but important, conceptual difference.
} 

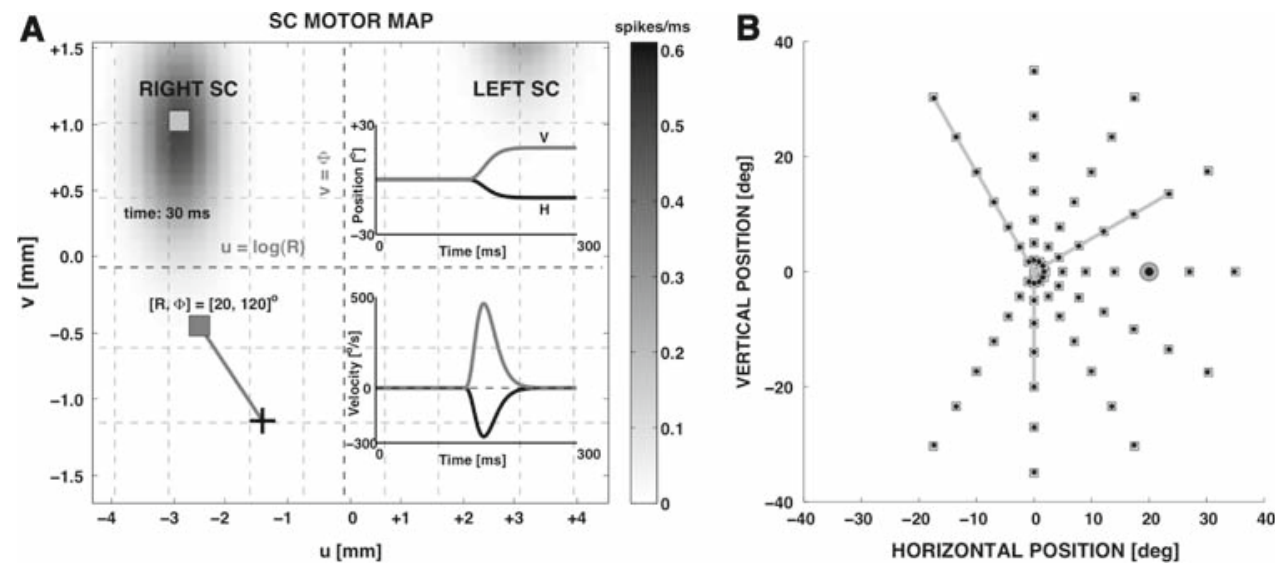

Fig. 9 a Snapshot of the model's activity in the motor map for a leftupward saccade at $[R, \Phi]=[20,120]$ deg. Insets in this panel show horizontal and vertical eye-position traces (top-right), eye-velocity profiles (bottom-right; note component stretching), and the associated 2D trajectory (bottom-left). The activity snapshot was taken at the moment of maximum firing of the gamma burst activity profiles ( $30 \mathrm{~ms}$ after burst onset). Note that activity is divided across the two motor maps. Grid superimposed on the anatomical $[\mathrm{u}, \mathrm{v}]$ coordinates shows iso-amplitude (running vertical, at $\mathrm{R}=0,2,5,20,50 \mathrm{deg}$ ) and iso-direction (running horizontal, $\Phi=-90,-60,-30,0,30,60,90 \mathrm{deg}$ ) lines of the motor map (Eq. 11). The $\mathrm{u}=0$ line in the center separates the two colliculi. b Targets (squares) and model saccade endpoints (black dots) for 63 locations. The target at $(20,0)$ deg (highlighted) was used to tune the model's weighting constant to $\eta=0.00039$. Three example trajectories are also shown (gray lines)
Fig. 10 Traces of eye position $\mathbf{a}$ and eye velocity $\mathbf{b}$ for simulated horizontal saccades to targets at $\mathrm{R}=[2,5,9,14,27$, 35] deg eccentricity. Note the asymmetric shape of the velocity profiles, which is due to a fixed acceleration time (determined by $e \cdot T_{0}=30 \mathrm{~ms}$, Eq. 14). The main sequence for these saccades is shown in the insets of panel $\mathbf{b}$
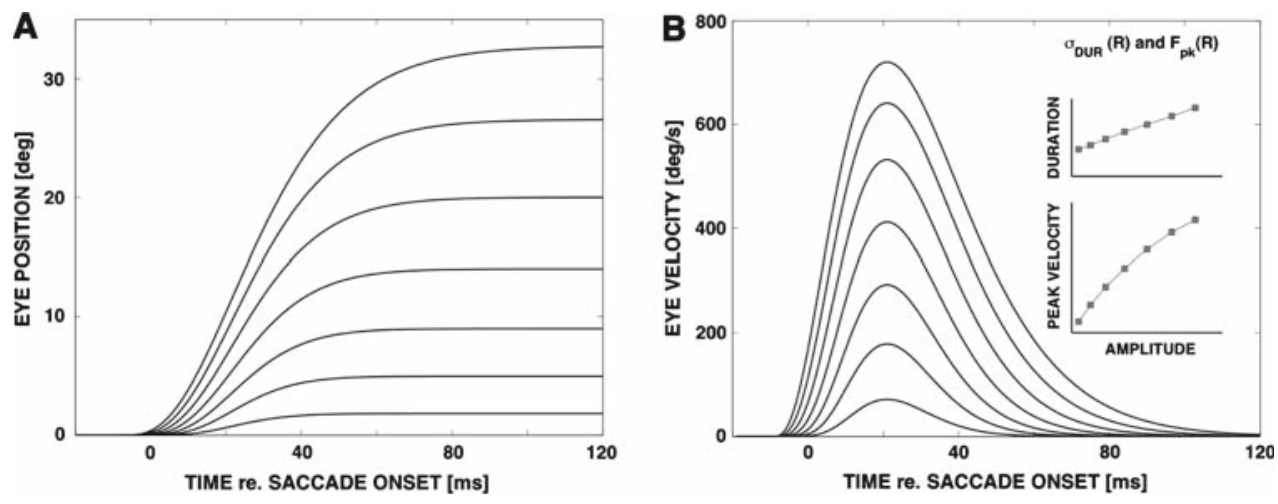

then tend to have shorter saccade-related bursts, with a higher peak-firing rate, than cells in more caudal areas of the motor map, while at the same time keeping the number of spikes for the optimal saccade constant. Figure 8 shows these relations, as they were used in our model simulations. We took $\sigma_{0}=3 \mathrm{~ms}, F_{0}=800 \mathrm{spikes} / \mathrm{s}$, and $\beta=0.07 \mathrm{~ms} / \mathrm{deg}$.

Figure $8 \mathrm{~b}$ shows the temporal activity profiles of the most active cell in the recruited population for a number of different saccade amplitudes. Note the drop in the cell's peak firing rate and the increase in burst duration, keeping the total number at $N_{0}=19$ spikes. These burst properties resemble the measured burst profiles shown in Fig. 7b.

Figure 9 illustrates how the model generates saccades in different directions and with different amplitudes. In Fig. 9a the motor activity in the SC map can be seen at the time of maximum firing ( $30 \mathrm{~ms}$ after burst onset) for a saccade with an amplitude of $20 \mathrm{deg}$, and a direction of $120 \mathrm{deg}$ (left and upward). Note that the activity transgresses the vertical meridian (the horizontal boundary at $v=\pi / 2 \mathrm{~mm}$ ), as a small part of the population activity is also found in the left SC. This adheres to the 'gluing problem' (Van Opstal and Van Gisbergen 1989; Tabareau et al. 2007). The insets in this panel show the horizontal/vertical eye position traces, the horizontal/vertical eye velocity, and the straight spatial trajectory of the saccade. Figure $9 \mathrm{~b}$ shows that the model generates accurate saccades in all directions and across all amplitudes.

The introduction of an amplitude-dependent increase of a cell's burst duration, and a concomitant decrease in its peak firing rate (Eq. 15) had a dramatic effect on the kinematics of saccades. Yet, the spatial trajectories of oblique eye movements remained straight.

Figure. 10a now clearly demonstrates that the duration of saccades increases with their amplitude. Figure 10b shows the saccade velocity profiles generated by the model, as well as the main-sequence properties of these eye movements. The main sequence behaviour of the model now resembles 

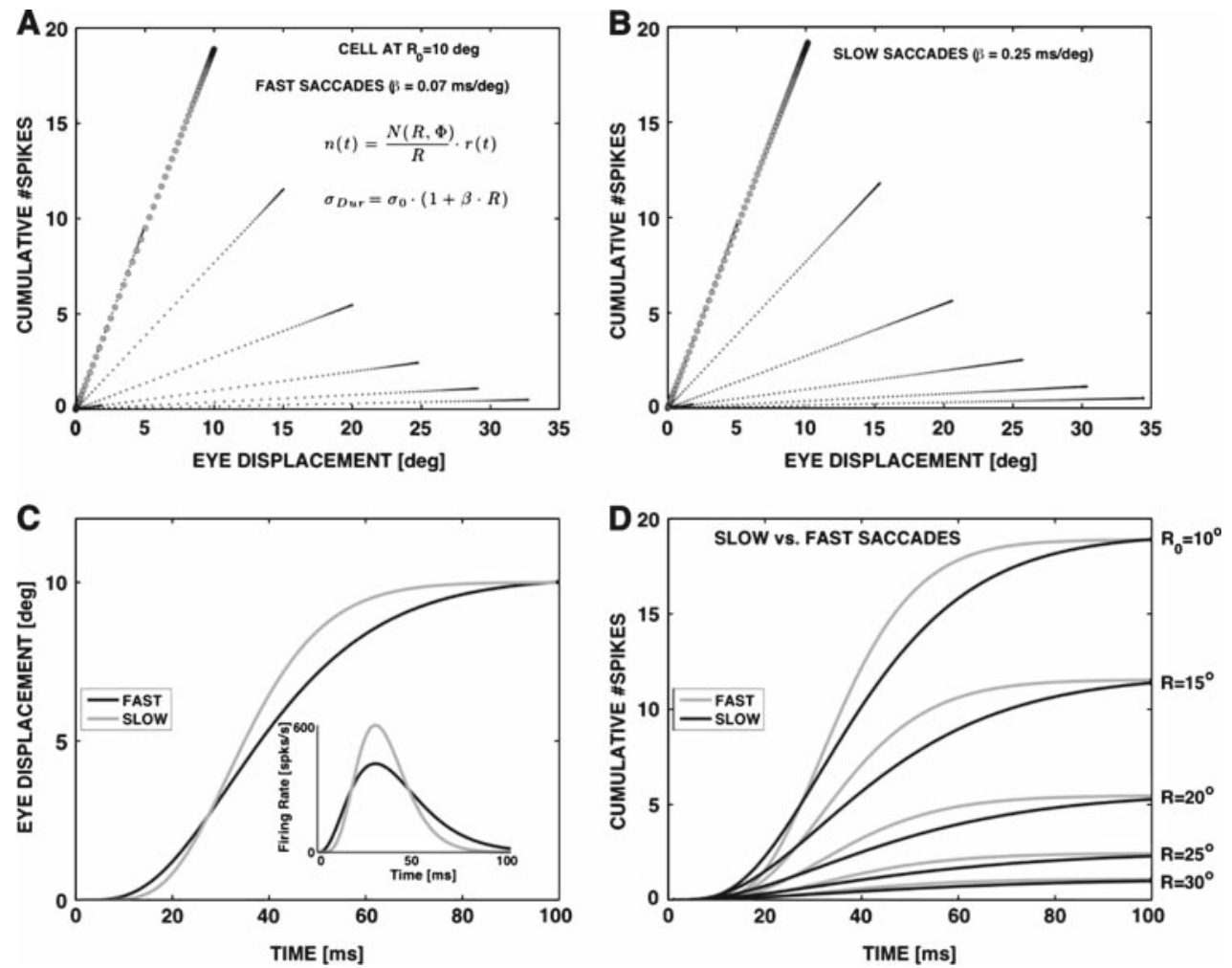

Fig. 11 a Cell activity during saccades into (gray symbols) and out of (black dots) the center of its movement field (centered at $[R, \Phi]=$ $[10,0] \mathrm{deg})$. Saccade amplitudes varied from $\mathrm{R}=[2,5,10,15,20$, $25,30,35] \mathrm{deg}$. The cumulative spike count is shown as function of the instantaneous eye displacement; individual dots correspond to time samples, not spikes. The cell's burst duration (and hence its peak mean firing rate) depended on saccade amplitude, $\mathrm{R}$ (Eq. 15, $\sigma_{0}=3 \mathrm{~ms}$; $\beta=0.07 \mathrm{~ms} / \mathrm{deg}$ ). The phase plots are straight, even for the saccades

with a low number of spikes, and their slopes depend systematically on the saccade vector (Eq. 10). b Same plots for slow saccades, simulated by setting $\beta=0.25 \mathrm{~ms} / \mathrm{deg}$. The phase plots of slow saccades are indistinguishable from the fast saccades in a. $\mathbf{c}$ Fast and slow eye displacements of $10 \mathrm{deg}$. The inset shows the corresponding burst profiles for this cell taking part in these saccades.d Cumulative spike counts of fast (gray) and slow (black) saccades follow different dynamics, because of different saccade kinematics

actual saccades quite well. In addition, the model also captures the duration-skewness relation of the velocity profiles. Thus, the mechanism described by Eq. 15, and illustrated by the recordings from a population of SC neurons in Fig. 7, may indeed underlie the nonlinear main-sequence properties of saccades, and constitute the mechanism by which the motor SC could act as a vectorial pulse generator.

An important prediction of the spike-vector ensemblecoding model is the linear relationship between the instantaneous cumulative number of spikes in the burst of a given $\mathrm{SC}$ cell, and the instantaneous displacement of the eye along the saccade trajectory (Eq. 10).

To illustrate this feature of the model, Fig. 11 shows these phase plots for a cell with an optimal saccade vector at $[10,0]$ deg. The cell response is shown for saccades of different amplitudes, each yielding a different number of spikes, and their own temporal profile, according to Eqs. 13-14. The phase plots in Fig. 11a are straight, even for the saccades with a low number of spikes in their burst. This property results from the assumption that burst durations and peak firing rates

depend systematically on the saccade vector rather than on the cell's location in the motor map. Figure 11b shows that the phase plots for very slow saccades of the same amplitudes (like those observed in blinking, and here simulated by setting $\beta=0.25 \mathrm{~ms} / \mathrm{deg}$ ) are indistinguishable from those of fast saccades. The actual saccades of the optimal amplitude are shown in Fig. 11c. The inset shows the corresponding burst profiles for the fast and slow saccade. The dynamics of the spike counts for the two conditions, however, are quite different, as is shown in Fig. 11d. All these properties of the model (captured by Eq. 10) are in line with the data as exemplified in Fig. 3 and reported in Goossens and Van Opstal (2006, their Figs. 4 and 5).

It is important to realise that straight phase trajectories, regardless of the saccade kinematics, are a consequence of the idea that the $\mathrm{SC}$ is directly involved in the motor program of the saccadic eye movement. This idea contrasts with other hypotheses, which assume that the SC cells encode only the desired vectorial displacement of the eye, and have no direct motor function (see Sect. 4). 


\section{Discussion}

In this paper, we have analysed our dynamic ensemblecoding model of the motor SC, in order to pinpoint the potential mechanism that may render the SC to act as a nonlinear vectorial pulse generator. We conclude, both on the basis of our recordings, illustrated in Fig. 7, and fully described in Goossens and Van Opstal (2006), as well as on the simulations with our model (Figs. 9, 10, 11), that the nonlinear kinematics of saccades are due to a gradient along the motor map of the burst properties of saccade-related cells. To summarise, saccade-related bursts can be characterised as follows:

(i) The total number of spikes for a cell's optimal saccade is fixed across the motor map (Fig. 7a, top).

(ii) For a given saccade, the total number of spikes of a cell is determined by the amplitude and direction of that saccade according to its classical movement field description (Ottes et al. 1986; Eqs. 3-4; Figs. 2 and 5).

(iii) The temporal distribution of the spikes can be described by gamma functions, with their peak firing rates at a fixed time relative to the saccade onset.

(iv) The amplitude and duration of the burst are determined by the cell's location within the motor map, such that the peak-firing rate decreases, and burst duration increases toward more caudal sites, while keeping the number of spikes constant. Without this mechanism, the dynamic ensemble-coding model of Fig. 4 behaves as a linear system (Fig. 6).

(v) The shape parameters of the burst are determined by saccade amplitude, such that for a given cell burst duration and skewness increase with the saccade amplitude for which it is recruited (Eq. 15; Fig. 7b).

\subsection{Why a nonlinear main sequence?}

We are well aware that we have grossly simplified the brainstem feedback loops by modelling them as linear systems (a gain with a feedback delay). The sole reason for this simplification, however, was to verify whether the spatial-temporal activity patterns in the motor SC could fully encode the saccade kinematics, without having to resort to nonlinear mechanisms like normalization of activity (e.g. center-of-gravity computation) or saturation of the brainstem pulse generator. Our previous study (Goossens and Van Opstal 2006) showed that the linear ensemble-coding model produced realistic saccades with compelling accuracy, while needing only three free linear parameters to generate the saccade kinematics across the entire repertoire of collicular activity patterns. We therefore decided to explore the properties of this scheme in greater detail.

In almost every model of the saccadic system, the brainstem local feedback loops are described by a saturating non- linearity that mimics the amplitude-peak velocity relationship (e.g. Van Gisbergen et al. 1981; Jürgens et al. 1981; Scudder 1988). The question is justified why the burst generator would contain this nonlinearity in the first place. It is not likely that it reflects a mere passive neural saturation, or neural fatigue, for several reasons: First, it has been shown that slow saccades (e.g. saccades to remembered targets in darkness) obey their own nonlinear main sequence (Smit et al. 1987). In addition, abducens oculomotor neurons reach firing rates that are comparable to those of the pontine burst neurons. Yet, models of the saccadic system invariantly employ linear transfer characteristics to describe these other types of neurons. Moreover, even though oculomotor neurons have nonlinear characteristics (as they are recruited beyond a threshold, essentially behaving as rectifiers), it is generally assumed that the output of the total neural population varies approximately linearly with changes in eye position.

Interestingly, a recent theoretical study by Harris and Wolpert (2006) suggested that the main-sequence properties of saccades could reflect an optimal control strategy of the system, as it has to cope with several conflicting constraints. The function of saccades is to redirect the fovea as fast and as accurately as possible to a peripheral target. However, the properties of internal noise within the system (assumed to increase with activity levels; this is e.g. visible in the movement field scans of Fig. 2), a low spatial resolution in the peripheral retina, and a penalty for overshooting the target (as commands then have to cross hemispheres), require a tradeoff between movement duration and accuracy. Their analysis indicated that the optimal trajectory to satisfy the constraints obeys the main-sequence relationships. On the basis of similar theoretical considerations, Tanaka et al. (2006) showed that the amplitude-duration relationship of saccades may follow from optimizing a trade-off between minimal movement duration and endpoint accuracy, by accounting both for inherent neural noise and the dynamics of the oculomotor plant.

Finally, earlier theoretical work by Harris (1995) had indicated that, given the main-sequence properties of saccades, another optimal strategy of the system to acquire the target on average as fast as possible would be to undershoot the target by about $10 \%$. Indeed, the phenomenon of saccadic undershoots is well established. Even when an experimental manipulation (e.g. magnifying lenses) produces accurate saccades on average, the system rapidly adapts to the new situation by developing undershoots (Henson 1978).

Thus, there appears to be a strong case against mere passive mechanisms, like neural saturation, that determine the motor control of saccades. Instead, the main sequence could result from a deliberate built-in optimal control strategy.

If so, one might wonder whether the optimal nonlinear controller could further benefit from embedding the mainsequence nonlinearity (and the undershooting strategy) at a 
vectorial encoding stage, rather than at the brainstem level of the horizontal and vertical saccade components. According to the common-source model of Van Gisbergen et al. (1985), an obvious benefit of a nonlinear vectorial pulse generator is that oblique saccade trajectories will automatically be straight without the need for an elaborate cross-coupling scheme (Smit et al. 1990).

Of course, straight saccade trajectories by themselves would be optimal in the sense that they constitute the shortest possible path between the start- and end points. Note that cross-coupling schemes such as proposed by Grossman and Robinson (1988), and later also by Nichols and Sparks (1996), could work as well as the common-source model to produce straight saccades. However, having (at least) two saturating nonlinearities in the brainstem leads to coupling coefficients that depend in a complicated way on the saccade amplitude and direction (Smit et al. 1990). In other words, generating straight saccades with coupled and unequal nonlinear horizontal and vertical burst generators requires an entire map of horizontal and vertical coupling coefficients. Moreover, these coefficients depend also on the saccade type (visually-evoked vs. remembered in darkness), which seems to be hardly an efficient and plausible network design.

In contrast, in the common source model component crosscoupling (Fig. 1) is an emergent property, which requires no additional tuning of the horizontal and vertical burst generators. Indeed, in our model (Fig. 4) the horizontal and vertical feedback loops are independent, yet the saccades are straight and the components show the appropriate amount of stretching. Moreover, fast and slow saccades are produced by the same mechanism, and therefore do not require separate sets of tuning parameters.

Note that the vectorial pulse-generator model does not imply that the horizontal and vertical saccade components always have equal durations. For example, when the gains of the horizontal and vertical feedback loops are different, saccade trajectories will curve toward the faster component. Patients suffering from Niemann-Pick type $\mathrm{C}$ disease appear to suffer from a deficit that selectively affects their vertical saccades (Rottach et al. 1997). It seems as if the gain of their vertical saccade generator may be decreased to only $10 \%$ of that of the horizontal system. Consequently, their oblique saccades are heavily curved toward the horizontal. The model of Fig. 4 can faithfully reproduce the responses of these patients, by simply letting $B_{V}=0.1 \cdot B_{H}$ (data not shown).

\subsection{Burst properties depend on the actual saccade vector}

In our simulations, the SC burst properties were assumed to depend on the actual saccade amplitude, $R$ (Eq. 15). Note that this is not a trivial assumption, as the burst duration and peak-firing rate could instead have depended on the cell's optimal saccade amplitude, $R_{0 k}$. In that case, the burst properties would have read:

$$
\begin{aligned}
\sigma_{\text {Dur }}\left(u_{k}\right) & =\sigma_{0} \cdot\left[1+\beta \cdot \exp \left(u_{k}\right)\right] \text { and } \\
F_{\mathrm{pk}}\left(u_{k}\right) & =\frac{F_{0}}{\sqrt{1+\beta \cdot \exp \left(u_{k}\right)}}
\end{aligned}
$$

where $u_{k}=\ln \left(R_{0 k}\right)$. Simulations with SC cells obeying Eq. 16 resulted in saccades that followed a virtually identical main sequence as with Eq. 15 (not shown)

Conceptually, however, Eqs. 15 and 16 describe quite different mechanisms to embody the main-sequence nonlinearity. According to Eq. 15 the temporal distribution of spikes in the burst, as well as the peak firing rate are determined by the actual saccade, $\vec{S}$, in which the cell is participating. Hence the temporal burst properties, as well as the number of spikes, are fully determined by the cell's input. Such a cell will fire a briefer and more intense burst of spikes when it takes part in saccades smaller than its optimum saccade, than when it participates in saccades that exceed its optimal amplitude, but for which it fires the same number of spikes. In this way, the distribution of firing rates within the active cell population is circular symmetric about its center in the motor map even though there is a rostral-to-caudal decrease in the firing rate of cells for their respective optimal vectors.

In the case of Eq. 16, however, the number of spikes is determined by the input, but the temporal firing properties of the cell are only determined by its location within the motor map, $\left[u_{k}, v_{k}\right]$, and hence by its optimal saccade, $\vec{S}_{0 k}$. Such a cell will fire identical spike trains for different saccades for which the number of spikes is the same. This would mean, however, that the distribution of firing rates within the active cell population would be skewed, since Eq. 16 implies that the rostral cells within this population have higher firing rates than caudal cells located at the same distance from the center. Our recordings support the scheme of Eq. 15 (not shown; manuscript in preparation).

A second difference between the two proposals is that the phase plots of $p(t)$ vs. $n_{k}(t)$ (see Fig. 11) will be straight for cells obeying Eq. 15, but they will be systematically curved for cells that are ruled by Eq. 16 (not shown). Note, however, that also in the latter case the resulting saccades will still be straight, and obey an almost identical nonlinear main sequence.

4.3 Center-of-gravity weighting combined with nonlinear feedback?

The experiments described in Goossens and Van Opstal (2006) suggested that the deep layers of the SC are involved in programming a saccadic motor command, and hence that the instantaneous activity (quantified by the cumulative number 


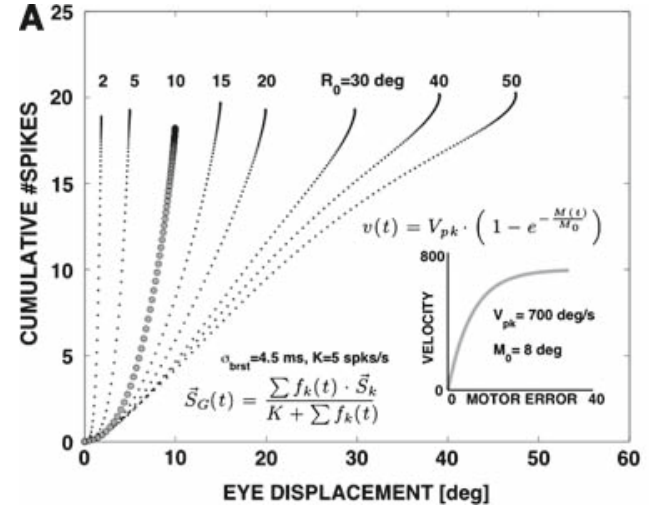

Fig. 12 Simulations with the center-of-gravity model (Eqs. 17-18). a Phase plots for the optimal saccade of different cells are not straight. Inset: brainstem burst generator nonlinearity. b The nonlinear model also fails to explain the invariant phase plots for fast (gray line) and slow

of spikes) relates linearly to the instantaneous eye displacement along the saccade trajectory (Eq. 10; Fig. 12).

However, alternative theories have been forwarded, that assign quite a different role to these cells: instead of encoding a motor command, they are thought to encode the saccade goal, imposed by the retinal target location (Port and Wurtz 2003; Krauzlis et al 2004; Walton et al. 2005). According to this theory, SC cells are not involved in generating the actual saccade trajectory and kinematics, but their weighted and scaled output (the center-of-gravity of the population, Eq. 7) represents the instantaneous desired eye-displacement signal toward the goal. This saccade goal corresponds to the eyecentered location of the target, and is then thought to drive mutually coupled nonlinear feedback loops in the brainstem (e.g. Port and Wurtz 2003; Walton et al. 2005).

We wondered, whether the observed relations between SC firing patterns and eye movements (like in Fig. 12) could also be obtained for such a scheme. Obviously, when the center-of-gravity computation is taken literally (Eq. 7), even a single spike in the motor map would already encode the final saccade goal, which is biologically implausible. Moreover, micro stimulation at low current intensities has been shown to yield saccades with smaller-than-optimal amplitudes (Van Opstal et al. 1990), which cannot be explained by an ideal center-of-gravity computation. A small change in the formulation of Eq. 7, however, could capture this phenomenon. Suppose, that the output of the SC yields a dynamic estimate of the saccade goal according to:

$\vec{S}(t)=\frac{\sum_{k \in \text { Pop }} f_{k}(t) \cdot \vec{S}_{0 k}}{K+\sum_{k \in \text { Pop }} f_{k}(t)}$

in which $f_{k}(t)$ is a cell's instantaneous firing rate, given by Eq. $13, \vec{S}_{0 k}$ is the cell's optimal saccade vector, and $K$ (in spikes/s) is a fixed constant. As burst activity profiles we

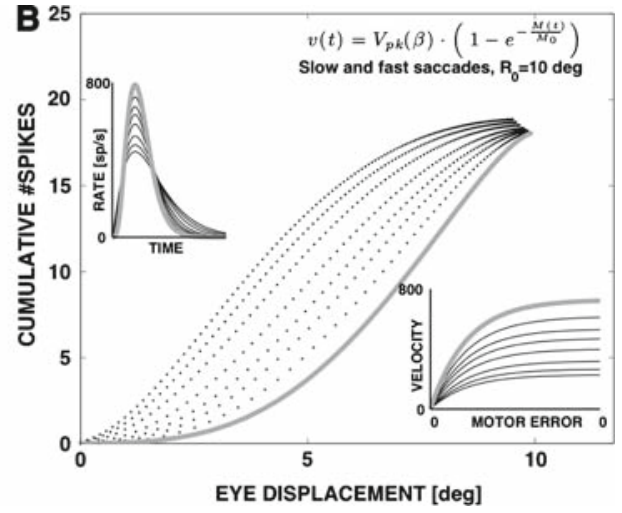

(dotted, black) saccades, here illustrated for the cell at the $\mathrm{R}=10 \mathrm{deg}$ site. Insets: gamma bursts for fast and slow $10 \mathrm{deg}$ saccades (left); dynamic burst generator nonlinearity (right). See text for explanation

took gamma bursts with a fixed duration, by setting $\sigma_{\text {Dur }}=$ $\sigma_{0}=4.5 \mathrm{~ms}$ (i.e. no implicit main-sequence properties in burst duration and peak firing rate like in Fig. 8, Eq. 15). When the total activity in the population is low, $K$ dominates the numerator and the desired saccade vector will be small. When the activity is high, $K$ may be neglected, and Eq. 17 approaches the center of gravity of the population.

A similar model has been proposed earlier (Van Opstal and Van Gisbergen 1989) to explain the results of micro stimulation and saccade weighted averaging to double stimuli.

The dynamic goal specified by Eq. 17 serves as the input to the nonlinear local feedback model of the brainstem proposed by Jürgens et al. (1981) and recently applied by Walton et al. (2005). The kinematic nonlinearity determines the horizontal and vertical eye-velocity components by:

$$
\begin{aligned}
\dot{e}_{H, V}(t) & =V_{\mathrm{pk}} \cdot\left[1-\exp \left(-\frac{M_{H, V}(t)}{M_{0}}\right)\right] \text { and } \\
M_{H, V}(t) & =S_{H, V}(t)-\int_{0}^{t} \dot{e}_{H, V}(\tau) d \tau
\end{aligned}
$$

where $M_{H, V}(t)$ is the dynamic motor error for the horizontal and vertical eye-movement components, given by the difference between the desired horizontal/vertical eye displacement of the goal, and the actual horizontal/vertical eye displacement since the saccade onset, encoded by resettable integrators in the feedback loop. Parameters $V_{\mathrm{pk}}$ and $M_{0}$ determine the amplitude-peak velocity nonlinearity of the main sequence. Typical values are $V_{p k}=700 \mathrm{deg} / \mathrm{s}$ and $M_{0}=8 \mathrm{deg}$. To obtain straight oblique saccades and component stretching, the horizontal and vertical burst generators would need to be coupled (see Sect. 1 and above), but we have not incorporated this feature here. Instead, we limited the simulations to horizontal saccades. 
Figure 12a shows the results of simulations with this model for cells with different optimal saccade amplitudes, ranging from $R_{0}=2$ to $50 \mathrm{deg}$. The burst duration of the SC cells was kept constant at $\sigma_{\text {Dur }}=4.5 \mathrm{~ms}$, and $K=5$ spikes $/ \mathrm{s}$. Note that although all curves increase monotonically, and reach approximately the same number of spikes for the optimal saccade, they are clearly not straight (cf. Fig. 11a).

The experimental data of Goossens and Van Opstal (2006) indicated that the phase relationships are also preserved for strongly perturbed, slow saccadic eye movements (e.g. Fig. 11b). To test the nonlinear vector-averaging model for this property we simulated lower firing rates in the SC for a site encoding a $10 \mathrm{deg}$ rightward saccade, by varying burstduration parameter $\beta$ (Eq. 15) between 0.01 and $0.25 \mathrm{~ms} / \mathrm{deg}$, where high values for $\beta$ correspond with low SC firing rates. Since the number of spikes in the bursts was kept constant between 18 and 20 spikes (in line with the experimental data, and Fig. 7a, top), the burst durations increased accordingly, as the peak-firing rates dropped (Fig. 12b, left-hand inset). Note that without any changes in the properties of the brainstem burst generator, saccades would maintain the same kinematics regardless of the SC burst profiles, as the dynamic goal signal of Eq. 17 is virtually unaffected by the SC firing rates. However, since it is well established that lower levels of SC activity tend to correlate with slower saccades (see Sect. 1), we incorporated the assumption of Sparks and coworkers (Lee et al. 1988; Nichols and Sparks 1996), that the asymptote of the burst generator ( $V_{\mathrm{pk}}$ in Eq. 18) co-varied with the SC firing rates according to $V_{\mathrm{pk}}(\beta)=V_{\mathrm{pk}} /(1+\beta)$ (Fig. 12b, inset lower right). However, despite this imposed kinematic relationship, the phase plot between the cumulative spike count of the SC cell and the actual eye displacement was not invariant to the temporal changes in the SC burst.

To summarise, the vector averaging scheme cannot capture the full repertoire of SC firing rate versus eye movement correlations, simply because that model denies a direct collicular role in motor control.

\subsection{Conclusion}

From the simulations in this paper, we conclude that the tight relationship between SC firing rates and saccadic eye displacement for both fast and very slow blink-perturbed saccades can only be explained if the nonlinear main sequence is embedded in the SC spatial-temporal activity patterns. We believe that our recordings, exemplified by the data in Figs. 3 and 8 , together with these simulations indicate that the saccade-related burst in the SC specifies a motor command, rather than the spatial goal for the upcoming saccade.

The mechanism by which the SC incorporates the main sequence, and hence acts as a nonlinear vectorial pulse generator, appears to be by a gradient in the burst properties across the motor map, from brief and intense in the rostral zone, to less intense and of longer duration in the caudal zone. Yet, the number of spikes for each cell's optimal saccade remains constant across the motor map and the distribution of firing rates within the active population follows a symmetrical, Gaussian profile.

Passive mechanisms like neural saturation or fatigue are not required to explain the observed results. Rather, the organization as described in this paper could result from an optimization principle that allows saccades to acquire the target as fast and as accurately as possible, given the conflicting constraints and noise within the system.

The question as to which mechanism ensures that for a given cell the number of spikes, and their temporal distribution, depends on the actual saccade amplitude, and hence on the neuronal input (Eqs. 13-15), will have to be explored in future studies.

Acknowledgements This research was funded by the Netherlands Organization for Scientific Research (NWO), project grants 805.05.003 (ALW/VICI, AJVO), and 864.06.005 (ALW/VIDI, HHLMG), the Radboud University Nijmegen (AJVO) and by the Radboud University Nijmegen Medical Center (HHLMG).

Open Access This article is distributed under the terms of the Creative Commons Attribution Noncommercial License which permits any noncommercial use, distribution, and reproduction in any medium, provided the original author(s) and source are credited.

\section{References}

Arai K, Keller EL (2005) A model of the saccade-generating system that accounts for trajectory variations produced by competing visual stimuli. Biol Cybern 92:21-37

Bahill AT, Clark MR, Stark L (1977) The main sequence, a tool for studying human eye movements. Math Biosci 24:191-204

Berthoz A, Grantyn A, Droulez J (1986) Some collicular efferent neurons code saccadic eye velocity. Neurosc Lett 72:289-294

Crawford JD, Guitton D (1997) Visual-motor transformations required for accurate and kinematically correct saccades. J Neurophysiol 78:1447-1467

Evinger C, Kaneko CR, Fuchs AF (1981) Oblique saccadic eye movements of the cat. Exp Brain Res 41:370-379

Goossens HHLM, Van Opstal AJ (2000) Blink-perturbed saccades in monkey. I. Behavioral analysis. J Neurophysiol 83:3411-3429

Goossens HHLM, Van Opstal AJ (2000) Blink-perturbed saccades in monkey. II. Superior colliculus activity. J Neurophysiol 83:3430 3452

Goossens HHLM, Van Opstal AJ (2006) Dynamic ensemble coding in the monkey superior colliculus. J Neurophysiol 95:2326-2341

Grossman GE, Robinson DA (1988) Ambivalence in modeling oblique saccades. Biol Cybern 58:13-18

Harris CM (1995) Does saccadic undershoot minimize saccadic flighttime? A Monte Carlo study. Vis Res 35:691-701

Harris CM, Wolpert DM (2006) The main sequence of saccades optimizes speed-accuracy trade-off. Biol Cybern 95:21-29

Henn V, Cohen B (1976) Coding of information about rapid eye movements in the pontine reticular formation of alert monkeys. Exp Brain Res 108:307-325

Henson DB (1978) Corrective saccades: effects of altering visual feedback. Vis Res 18:63-67 
Jürgens R, Becker W, Kornhuber HH (1981) Natural and drug-induced variations of velocity and duration of human saccadic eye movements: evidence for a control of the neural pulse generator by local feedback. Biol Cybern 39:87-96

Kato R, Grantyn A, Dalezios Y, Moschovakis AK (2006) The local loop of the saccadic system closes downstream of the superior colliculus. Neuroscience 143:319-337

Keller EL, Edelman JA (1994) Use of interrupted saccade paradigm to study spatial and temporal dynamics of saccadic burst cells in superior colliculus in monkey. J Neurophysiol 72:2754-2770

Klier EM, Wang H, Crawford JD (2001) The superior colliculus encodes gaze commands in retinal coordinates. Nature Neurosci 4:627-632

Krauzlis RJ, Liston D, Carello CD (2004) Target selection and the superior colliculus: choices and hypotheses. Vis Res 44:1445-1451

Lee C, Rohrer WH, Sparks DL (1988) Population coding of saccadic eye movement by neurons in the superior colliculus. Nature 332: 357-360

Luschei ES, Fuchs AF (1972) Activity of brainstem neurons during eye movements of alert monkeys. J Neurophysiol 35:445-461

Matsuo S, Bergeron A, Guitton D (2004) Evidence for gaze feedback to the cat superior colliculus:discharges reflect gaze trajectory perturbations. J Neurosci 24:2760-2773

Munoz DP, Waitzman DM, Wurtz RH (1996) Activity of neurons in monkey superior colliculus during interrupted saccades. J Neurophysiol 75:2562-2580

McIlwain JT (1982) Lateral spread of neural excitation during microstimulation in intermediate gray layer of cat's superior colliculus. J Neurophysiol 47:167-178

Nichols MJ, Sparks DL (1996) Component stretching during oblique stimulation-evoked saccades: the role of the superior colliculus. J Neurophysiol 76:582-600

Ottes FP, Van Gisbergen JAM, Eggermont JJ (1986) Visuomotor fields of the superior colliculus: a quantitative model. Vis Res 26:857873

Port NL, Wurtz RH (2003) Sequential activity os simultaneously recorded neurons in the superior colliculus during curved saccades. J. Neurophysiol 90:1887-1903

Quaia C, Lefèvre P, Optican LM (1999) Model of the control of saccades by superior colliculus and cerebellum. J Neurophysiol 82:999-1018

Robinson DA (1972) Eye movements evoked by collicular stimulation in the alert monkey. Vis Res 12:1795-1808

Rottach KG, Von Maydell RD, Das VE, Zivotovsky AZ, Discenna AO, Gordon JL, Landis DMD, Leigh RJ (1997) Evidence for independent feedback control of horizontal and vertical saccades from Niemann-Pick Type C disease. Vis Res 37:3627-3638

Scudder CA (1988) A new local feedback model of the saccadic burst generator. J Neurophysiol 59:1455-1475

Smit AC, Van Gisbergen JAM, Cools AR (1987) A parametric analysis of human saccades in different experimental paradigms. Vis Res $27: 1745-1762$
Smit AC, Van Opstal AJ, Van Gisbergen JAM (1990) Component stretching in fast and slow oblique saccades in the human. Exp Brain Res 81:325-334

Soetedjo R, Kaneko CRS, Fuchs AF (2002) Evidence that the superior colliculus participates in the feedback control of saccadic eye movements. J Neurophysiol 87:679-695

Sparks DL, Holland R, Guthrie BL (1976) Size and distribution of movement fields in the monkey superior colliculus. Brain Res 113:21-34

Stanford TR, Freedman EG, Sparks DL (1996) Site and parameters of microstimulation: evidence for independent effects on the properties of saccades evoked from the primate superior colliculus. J Neurophysiol 76:3360-3381

Tanaka H, Krakauer JW, Qian N (2006) An optimization principle for determining movement duration. J Neurophysiol 95:3875-3886

Tabareau N, Bennequin D, Berthoz A, Slotine JJ, Girard B (2007) Geometry of the superior colliculus mapping and efficient oculomotor computation. Biol Cybern 97:279-292

Van Gisbergen JAM, Van Opstal AJ (1989) Models. The neuro biology of saccadic eye movements. In: Wurtz RH, Goldberg ME (eds) Reviews of Oculomotor Research. Elsevier, Amsterdam, pp 69-98

Van Gisbergen JAM, Robinson DA, Gielen S (1981) A quantiative analysis of generation of saccadic eye movements by burst neurons. J Neurophysiol 45:417-442

Van Gisbergen JAM, Van Opstal AJ, Schoenmakers JJM (1985) Experimental test of two models for the generation of oblique saccades. Exp Brain Res 57:321-336

Van Gisbergen JAM, Van Opstal AJ, Tax AAM (1987) Collicular ensemble coding of saccades based on vector summation. Neuroscience 21:541-555

Van Opstal AJ, Van Gisbergen JAM (1987) Skewness of saccadic velocity profiles: a unifying parameter for normal and slow saccades. Vis Res 27:731-745

Van Opstal AJ, Van Gisbergen JAM, Smit AC (1990) Comparison of saccades evoked by visual stimulation and collicular electrical stimulation in the alert monkey. Exp Brain Res 79:299-312

Van Opstal AJ, Van Gisbergen JAM (1989) A nonlinear model for collicular spatial interactions underlying the metrical properties of electrically elicited saccades. Biol Cybern 60:171-183

Waitzman DM, Ma TP, Optican LM, Wurtz RH (1991) Superior colliculus neurons mediate the dynamic characteristics of saccades. J Neurophysiol 66:1716-1737

Walton MM, Sparks DL, Gandhi NJ (2005) Simulations of saccade curvature by models that place superior colliculus upstream from the local feedback loop. J Neurophysiol 93:2354-2358

Westheimer G (1954) Mechanism of saccadic eye movements. Arch Ophthalmol 52:710-724 